\title{
Reduced-intensity versus Myeloablative Conditioning Regimens for Younger Adults with Acute Myeloid Leukemia and Myelodysplastic Syndrome: A systematic review and meta-analysis
}

\author{
Shengling $\mathrm{Ma}^{1^{*}}$, Wei Shi ${ }^{1,2^{*}}$, Ziying $\mathrm{Li}^{1}$, Liang Tang ${ }^{1}$, Huafang Wang ${ }^{1}$, Linghui $\mathrm{Xia}^{1}, \mathrm{Yu} \mathrm{Hu}^{1 凶}$ \\ 1. Institute of Hematology, Union Hospital, Tongii Medical College, Huazhong University of Science and Technology, Wuhan, China. \\ 2. Fred Hutchinson Cancer Research Center, Seattle, WA 98109-1024, USA. \\ *These authors contributed equally to this work.
}

$\square$ Corresponding author: Yu Hu, Address: Institute of Hematology, Union Hospital, Tongji Medical College, Huazhong University of Science and Technology, Wuhan 430022, China; Tel: +86-27-85726335; Fax: +86-27-85726343; E-mail: dr_huyu@126.com.

(c) The author(s). This is an open access article distributed under the terms of the Creative Commons Attribution License (https://creativecommons.org/licenses/by/4.0/). See http://ivyspring.com/terms for full terms and conditions.

Received: 2020.03.17; Accepted: 2020.06.15; Published: 2020.07.06

\begin{abstract}
Background: Historically, reduced-intensity conditioning (RIC) was recommended to be performed for older patients who were considered ineligible for myeloablative conditioning (MAC) before allogeneic hematopoietic stem cell transplantation (allo-HSCT). However, the evidence regarding the optimal conditioning intensity in younger patients with AML or MDS is weak and contradictory.

Methods: PubMed, Medline, Embase, and other online sources were searched from the initial period to February 25, 2020. Odds ratios and 95\% confidence intervals were calculated to estimate pooling effects.

Results: Four randomized controlled trials (RCTs) about conditioning intensity involving 633 patients were included. There were no significant differences of $1 / 2 / 4 / 5$ years progression-free survival (PFS) and relapse incidence (RI) between two conditioning intensities. Overall survival (OS) was similar at 1/2/4 years, but patients receiving RIC had a higher OS at 5 years. Additionally, RIC were associated with lower non-relapse mortality, less grade II-IV and grade III-IV acute graft-versus-host disease (GVHD), and lower incidence of chronic GVHD compared with MAC regimens. Subgroup analysis showed similar OS and RI for AML patients, and there was a trend towards lower NRM and grade II-IV aGVHD in RIC group. Available data for MDS indicated that OS, PFS, and RI were comparable. For intermediate-risk patients, there was no evidence that RIC is inferior to MAC. However, for high-risk patients, MAC tends to perform better.

Conclusions: Based on the above results, it might be concluded that RIC is a feasible treatment option for adults with AML or MDS younger than 66 years, particularly those with intermediate-risk disease. Future RCTs incorporating of risk stratifications are warranted to guide the optimal decision under certain conditions.
\end{abstract}

Key words: myeloablative, stem cell, acute myeloid leukemia

\section{Introduction}

Allogeneic hematopoietic stem cell transplantation (allo-HSCT) is the backbone therapy for patients with acute myeloid leukemia (AML) or myelodysplastic syndromes (MDS) [1]. Historically, reduced-intensity conditioning (RIC) regimens prior to allo-HSCT were typically the strategy in older patients or younger patients with complicated comorbidities that are intolerable of myeloablative conditioning (MAC) regimens. However, ongoing interest has been shown in RIC regimens as 
consolidation therapy due to lower NRM and comparable survival, which were reported to be performed in two-thirds of transplanted patients in the past two decades [2-4]. A series of retrospective studies have drawn contradictory conclusions about the optimum conditioning intensity of younger AML/MDS patients [5-11]. The variability in age, performance status, and different comorbidities interfere with the ability to isolate the effects of conditioning intensities on outcomes.

To date, RIC allogeneic HSCT is a feasible treatment option for AML patients aged 60 years or older in the most-updated NCCN clinical practice guidelines (V2.2020) [12] still, for younger patients, there is a lack of high-level evidence for the use of RIC regimens [13]. Therefore, we conducted a timely systematic review and meta-analysis of recent randomized controlled trials (RCTs) to provide quantitative evidence on the overall efficacy and toxicity of RIC versus MAC in younger adults with AML or MDS.

\section{Methods}

\section{Searching strategy}

According to the recommendations of the Cochrane Collaboration [14, 15], we retrieved articles from PubMed, Medline, Embase, and Google scholar from their inception until February 25, 2020. Abstracts from the conference proceedings, ongoing and unpublished trials in https://www.clinicaltrials.gov and the bibliographies of other relevant reviews were also manually identified.

We used a combination of corresponding keywords: "allogeneic hematopoietic stem cell transplantation", "reduced-intensity", "myeloablative", "acute myeloid leukemia" and "myelodysplastic syndromes".

\section{Definitions of outcomes}

The primary outcomes included overall survival (OS) and progression-free survival (PFS). The secondary outcomes consisted of non-relapse mortality (NRM), relapse incidence (RI), incidence of acute graft-versus-host disease (aGVHD), and chronic GVHD (cGVHD). The concept of PFS covered relapsefree survival, disease-free survival and leukemia-free survival. Similarly, the concept of NRM included transplant-related mortality (TRM).

\section{Selection criteria and study selection}

We included all comparative studies that met the following criteria: (1) RCTs included AML/MDS patients in complete remission who received either RIC or MAC; (2) the study reported sufficient patient demographics and outcomes.
To perform a systematic and comprehensive assessment, we combined all the multiple publications. Two investigators (Ma and Shi) selected the studies independently. Any discrepancy regarding eligibility was solved by consulting the senior investigator $(\mathrm{Hu})$.

\section{Data extraction and quality assessment}

Two investigators (Ma, Shi) independently extracted the following information from each eligible study: characteristics (first author, year of the last publication, country, sample size, follow-up time, participant numbers of each group, period of enrollment, recruitment period), participant characteristics(sex, the median age at enrollment, diagnosis), intervention details (conditioning regimens, transplantation details) and outcomes. We contacted all corresponding authors for insufficient data in the articles. We assessed the methodological bias of the included trials by the Cochrane Collaboration's tool [16]. The senior author $(\mathrm{Hu})$ assessed all disagreements in bias.

\section{Data synthesis}

Discontinuous data were pooled and calculated as odds ratios (OR) by using a $95 \%$ confidence interval (CI). If the publications did not provide data at a particular time point but curves, we used the methods reported by Tierney [17] and Parmer [18].

The summary effect estimates of OR for individual RCTs are indicated by solid squares, with a size proportional to the sample size and the number of events. Horizontal lines indicate 95\% confidence interval. The diamonds indicate CIs for pooled effects, with the size of the box relating to the weight of the study. We assessed the heterogeneity among studies by the $\mathrm{Q}$ test $(\mathrm{P}<0.05$ to be indicative of statistically significant) and Higgins $\mathrm{I}^{2}$ parameters (25-50\%, 50-75\%, and $>75 \%$ were divided into low, moderate, and high heterogeneity) [19]. As indicated by DerSimonian and Laird [20], if $\mathrm{I}^{2} \geq 50 \%$, we chose the random-effect model; otherwise, we used the fixed-effect model. In the sensitivity analysis, the origin of heterogeneity was evaluated by repeating the meta-analysis after removing one study at a time. Moreover, we conducted subgroup analyses by diagnoses with the data available in included RCTs.

All statistical analyses were performed using Review Manager 5.3. All tests were 2-sided, and $\mathrm{P}<0.05$ was considered statistically significant.

\section{Results}

\section{Literature search and study characteristics}

Figure 1 summarized the processes of study selection. After two rounds of careful screening and 
hand searches, 4 RCTs [21-24] reported in 7 publications were initially selected for this systematic review, including two abstract proceedings presenting long term follow-ups [25, 26] and an updated report [27] of the previously published one $[22,23]$. We excluded two non-randomized prospective studies $[28,29]$.

Table 1 depicts the main characteristics of the included RCTs. Among them, three studies were performed in Europe and one in the United States. All included studies were published from 2013 to 2018. Altogether, 633 patients were randomized to be treated with either RIC (319 [50.4\%]) or MAC (314 [49.6\%]). Four hundred fifty-four patients were diagnosed as AML, 169 were MDS, and the others were CML or missing. Three RCTs [21-23] applied a uniform conditioning regimen in each arm and a uniform GVHD prophylaxis regimen, whereas several choices were available in the trial of Scott et al. [24]. The reported enrolled patients ranged in age from 18 to 66 years, and the median ages of each eligible study varied from 44 to 54.8 .

\section{Risk of bias}

According to the Cochrane Handbook, the overall bias risk of these RCTs was judged to be low to moderate (Figure 2). All RCTs reported the randomization details. Due to the special nature of transplantation, none of the four studies used double-blind methods. The outcomes of 2 trials [24, 27] were assessed with a low risk of bias, and missing data were described [24, 27]. All four studies were free of selective outcomes reporting. Four trials were judged to have a low risk of other bias.

\section{Effect on overall survival}

OS rates were all extractable from the survival curves in 4 RCTs (four for OS at 1 and 2 years, three for 4 [26, 27] and 5 years [27], Figure 3A). Specifically, two of them were from the updated abstracts $[25,26]$. The rates of OS at 1,2 and 4 years were similar between patients who received RIC and those who received $\mathrm{MAC}(\mathrm{OR}=0.78,95 \%$ CI $0.27-2.24, \mathrm{P}=0.65$, $\mathrm{I}^{2}=84 \%$; OR=1.27, 95\% CI $0.68-2.38, \mathrm{P}=0.45, \mathrm{I}^{2}=64 \%$; $\mathrm{OR}=1.01,95 \%$ CI $\left.0.42-2.38, \mathrm{P}=0.99, \mathrm{I}^{2}=77 \%\right)$. However, OS was statistically significantly better with RIC instead of MAC at 5 years (OR=1.69, 95\% CI 1.10-2.59, $\left.\mathrm{P}=0.02, \mathrm{I}^{2}=0 \%\right)$, in line with the tendency in the second-largest RCT [27] that RIC achieved similar OS (61\% at 3 years, $60 \%$ at 10 years) but MAC resulted in significantly lower survival at 10 years (47\%) compared to that at 3 years (58\%). Three trials presented comparable OS at the end of the study except one [26], which reported better OS for MAC at 4 years (65\% vs. $49 \%, p=0.02)$. Accounting for the moderate and high heterogeneities in subgroups at 2 and 4 years, the $\mathrm{I}^{2}$ was valued $0 \%$ after repeating the meta-analysis removing Scott et al.'s study.

Table 1. Characteristics of studies included in the meta-analysis

\begin{tabular}{|c|c|c|c|c|c|c|c|c|c|}
\hline & & \multicolumn{2}{|c|}{ Bornhäuser $2018[23,27]$} & \multicolumn{2}{|c|}{ Kroger $2018[22,25]$} & \multicolumn{2}{|c|}{ Ringden 2013 [21] } & \multicolumn{2}{|l|}{ Scott $2020[24,26]$} \\
\hline \multicolumn{2}{|l|}{ Country } & \multicolumn{2}{|c|}{ Germany } & \multicolumn{2}{|c|}{ Germany } & \multicolumn{2}{|c|}{ Sweden } & \multicolumn{2}{|l|}{ USA } \\
\hline \multicolumn{2}{|l|}{ Number of centers } & \multicolumn{2}{|l|}{13} & \multicolumn{2}{|l|}{18} & \multicolumn{2}{|l|}{1} & \multicolumn{2}{|l|}{32} \\
\hline $\begin{array}{l}\text { Conditioning } \\
\text { regimen }\end{array}$ & & RIC & MAC & RIC & MAC & RIC & MAC & RIC & MAC \\
\hline \multicolumn{2}{|l|}{ Protocol } & $\begin{array}{l}\text { Flu } \\
\left(150 \mathrm{mg} / \mathrm{m}^{3}\right) \\
+ \text { TBI (8 Gy) }\end{array}$ & $\begin{array}{l}\text { TBI }(12 \mathrm{~Gy})+ \\
\text { Cy }(120 \mathrm{mg} / \mathrm{kg})\end{array}$ & $\begin{array}{l}\text { Flu } \\
\left(150 \mathrm{mg} / \mathrm{m}^{3}\right)+ \\
\text { Bu }(8 \mathrm{mg} / \mathrm{kg} \\
\text { orally or } 6.4 \\
\text { mg/kg i.v. })\end{array}$ & $\begin{array}{l}\mathrm{Bu}(16 \mathrm{mg} / \mathrm{kg} \\
\text { orally or } 12.8 \mathrm{mg} / \\
\mathrm{kg} \text { i.v. })^{+} \\
\mathrm{Cy}(120 \mathrm{mg} / \mathrm{kg})\end{array}$ & $\begin{array}{l}\text { Flu } \\
\left(180 \mathrm{mg} / \mathrm{m}^{3}\right)+ \\
\mathrm{Bu}(8 \mathrm{mg} / \mathrm{kg})\end{array}$ & $\begin{array}{l}\mathrm{Bu} \\
(16 \mathrm{mg} / \mathrm{kg}) \\
+\mathrm{Cy} \\
(120 \mathrm{mg} / \mathrm{kg})\end{array}$ & $\begin{array}{l}\text { Flu }(120 \text { to } \\
\left.180 \mathrm{mg} / \mathrm{m}^{2}\right)+\mathrm{Bu} \\
(\leq 8 \mathrm{mg} / \mathrm{kg} \text { orally } \\
\text { or } 6.4 \mathrm{mg} / \mathrm{kg} \text { i.v. }) \\
\text { FluMel }\end{array}$ & $\begin{array}{l}\text { Flu }\left(120-180 \mathrm{mg} / \mathrm{m}^{2}\right)+ \\
\mathrm{Bu}(16 \mathrm{mg} / \mathrm{kg} \text { orally or } \\
12.8 \mathrm{mg} / \mathrm{kg} \text { i.v. }) ; \\
\mathrm{Bu}+\mathrm{Cy}(120 \mathrm{mg} / \mathrm{kg}) \\
\text { TBI }(12-14.2 \mathrm{~Gy})+\mathrm{Cy} .\end{array}$ \\
\hline No. of cases & & 99 & 96 & 65 & 64 & 18 & 19 & 137 & 135 \\
\hline Male, $\%$ & & $43(43 \%)$ & $47(49 \%)$ & $38(59 \%)$ & $8(23 \%)$ & $12(63 \%)$ & $11(79 \%)$ & $67(49 \%)$ & $76(56 \%)$ \\
\hline $\begin{array}{l}\text { Median age } \\
\text { (range),y }\end{array}$ & & $44(18-60)$ & $45(18-60)$ & $51(22-63)$ & $50(19-64)$ & $46(26-61)$ & $45(22-58)$ & $54.8(21.9-65.9)$ & $54.8(21.9-66)$ \\
\hline Diagnosis & & \multicolumn{2}{|l|}{ AML } & \multicolumn{2}{|c|}{ MDS: 115; sAML: 12; missing: 2} & \multicolumn{2}{|c|}{ AML: 29; CML: 8} & \multicolumn{2}{|l|}{ AML: 218; MDS: 54} \\
\hline $\begin{array}{l}\text { GVHD } \\
\text { prophylaxis }\end{array}$ & & \multicolumn{2}{|l|}{ CsA / MTX } & \multicolumn{2}{|l|}{ CsA/MTX } & \multicolumn{2}{|l|}{ CsA/MTX } & \multicolumn{2}{|c|}{ CNI/MMF, CNI/MTX, Tac/Siro } \\
\hline \multirow[t]{3}{*}{ Donor type } & MRD & 59 & 58 & 16 & 17 & 7 & 7 & 58 & 57 \\
\hline & MUD & 28 & 24 & 38 & 36 & 11 & 12 & 58 & 66 \\
\hline & Other & 12 & 14 & 11 & 11 & 0 & 0 & 21 & 12 \\
\hline \multirow[t]{2}{*}{ Graft source } & PBSC & 90 & 90 & 59 & 61 & 17 & 16 & 123 & 127 \\
\hline & $\mathrm{BM}$ & 9 & 6 & 6 & 3 & 1 & 3 & 14 & 8 \\
\hline $\begin{array}{l}\text { Recuitment } \\
\text { period }\end{array}$ & & 2004-2009 & & 2004-2012 & & NR & & 2011-2014 & \\
\hline $\begin{array}{l}\text { Median follow-up } \\
\text { (range), month }\end{array}$ & & $\begin{array}{l}119 \\
(102-137)\end{array}$ & $\begin{array}{l}119 \\
(102-138)\end{array}$ & 72 & 75 & $40.8(6-104.4)$ & $\begin{array}{l}62.4 \\
(14.4-111.6)\end{array}$ & 50 & 50 \\
\hline
\end{tabular}




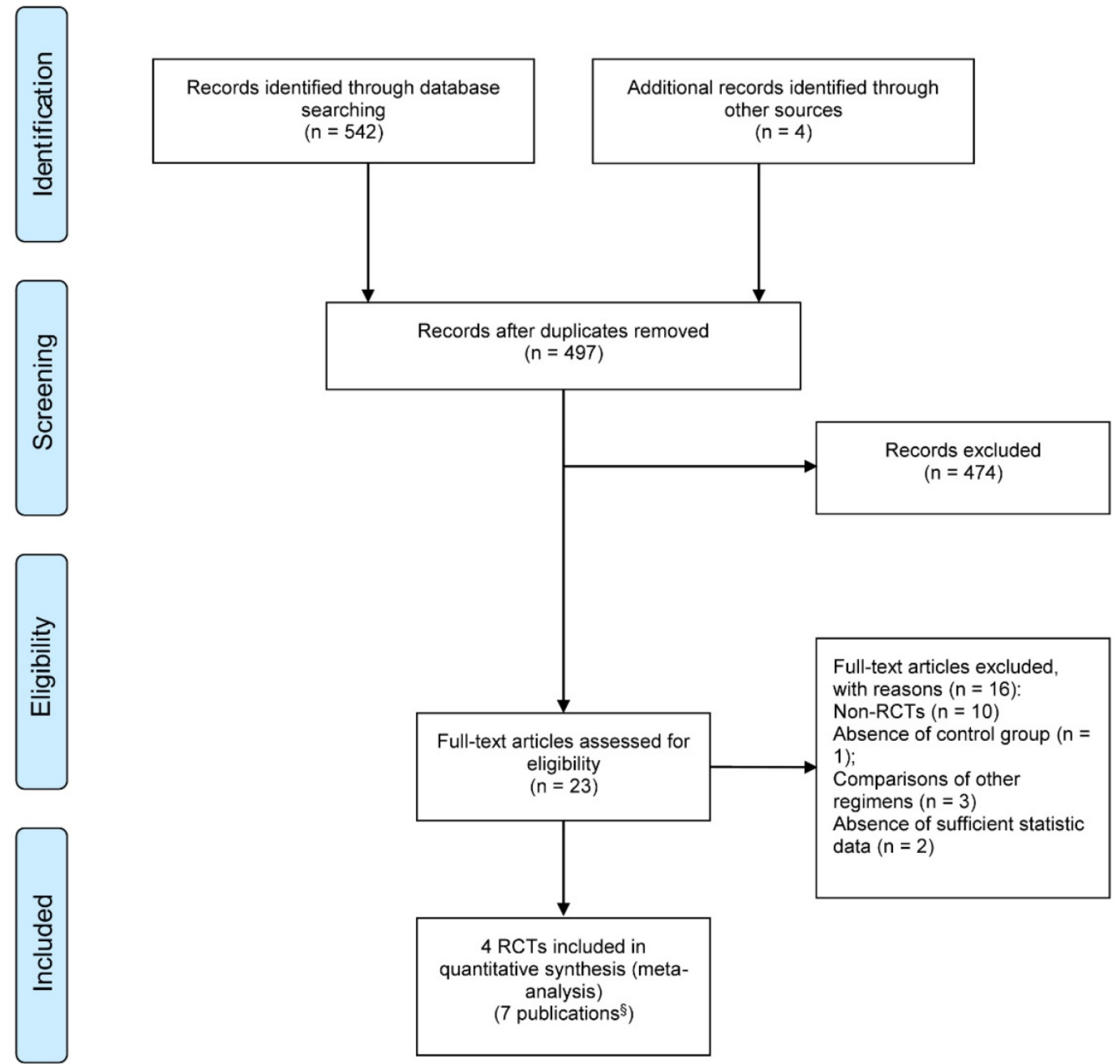

Figure 1. PRISMA flow diagram of study selection; § Includes 4 full text articles and 3 updated publications. RCT: randomized controlled trials.

\section{Effect on progression-free survival}

As shown in Figure 3B, PFS data, which comprised a total of 596 patients, were provided at 1 , 2 , and 4 years, while data from 361 patients were analyzed at 5 years. The pooled ORs were comparable: $0.71(95 \% \mathrm{CI} 0.28-1.80 ; \mathrm{P}=0.47)$ at 1 year, $0.81(95 \% \mathrm{CI} 0.34-1.92, \mathrm{P}=0.63)$ at 2 years, $0.83(95 \% \mathrm{CI}$ $0.35-1.98, \mathrm{P}=0.67)$ at 4 years and $1.30(95 \% \mathrm{CI} 0.85-1.99$, $\mathrm{P}=0.23$ ) at 5 years. Similar to OS, heterogeneity at 1,2 , 4 years $\left(\mathrm{I}^{2}=85 \%\right)$ disappeared when Scott et al.'s study was ruled out.

\section{Effect on non-relapse mortality}

All studies, including 633 patients, reported data on NRM at 1, 2, 5 years. As depicted in Figure 4A, the combined ORs for 1, 2, 5-year NRM were 0.45 (95\% CI $0.27-0.75 ; \mathrm{P}=0.002), 0.47$ (95\% CI $0.30-0.75 ; \mathrm{P}=0.001)$ and 0.48 (95\% CI 0.31-0.75; $\mathrm{P}=0.001$ ), respectively. Without heterogeneity in all subgroups $\left(\mathrm{I}^{2}=0 \%\right)$, additional subgroup or sensitivity analyses were unnecessary. Accordingly, there was strong evidence for the reduced incidence of NRM in the RIC intervention group.

\section{Effect on relapse incidence}

Pooled analysis of all studies presented no statistically difference between two interventions among different follow-up durations (1 year: 2.11, 95\% CI 0.67-6.67, $\mathrm{P}=0.20$; 2 years: $1.43,95 \%$ CI $0.43-4.70, \mathrm{P}=0.56$; 5 years: $1.27,95 \%$ CI $0.41-3.94$, $\mathrm{P}=0.68$; Figure $4 \mathrm{~B})$. While similar relapse risk was found in the other three trials, only one study [26] reported higher relapse incidence, contributing to the high heterogeneity in three subgroups $\left(\mathrm{I}^{2}=80 \%, 87 \%\right.$, $88 \%)$. 


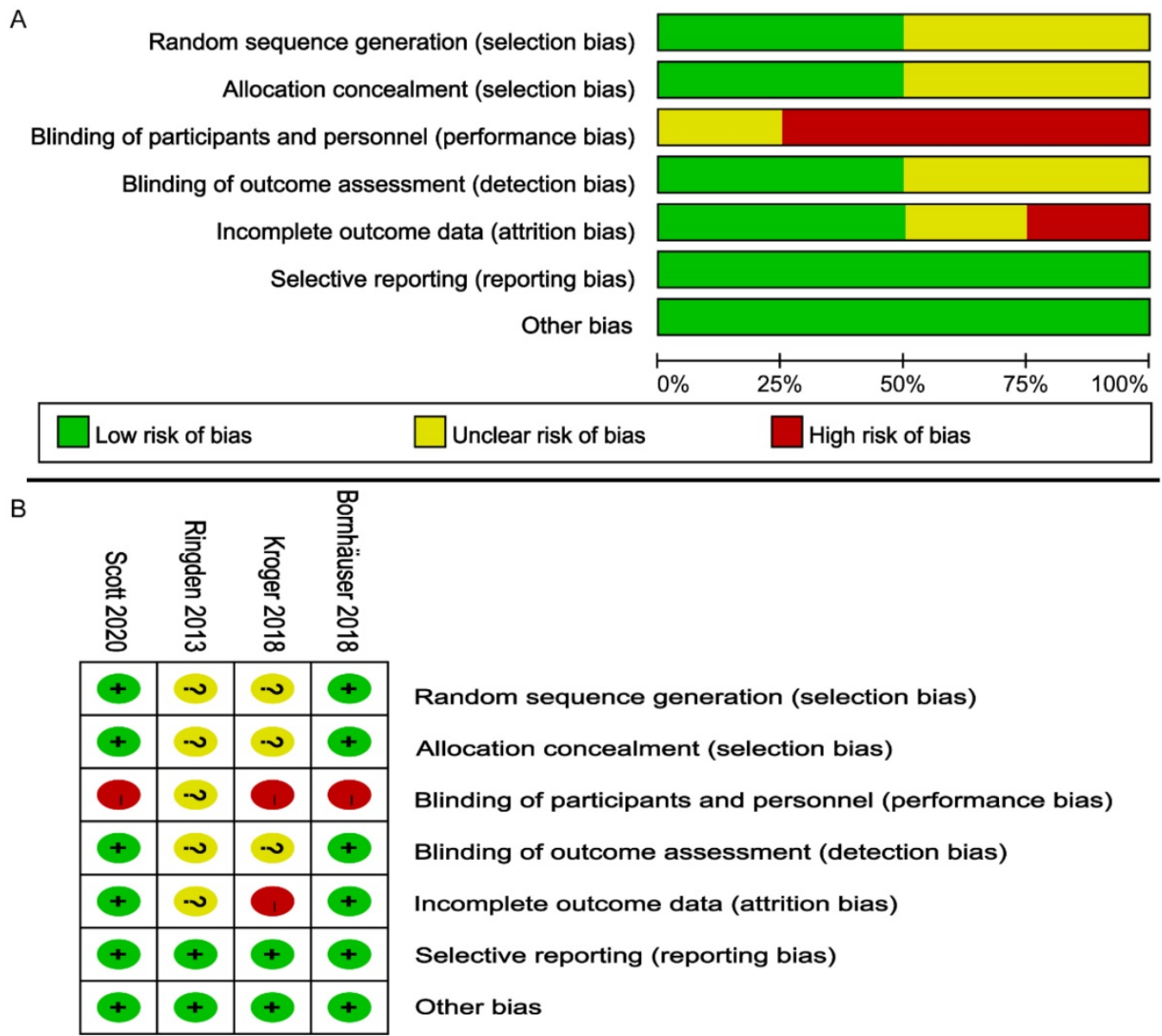

Figure 2. Risk of bias assessment on the included 4 RCTs; (A) Risk of bias graph; (B) Risk of bias summary.

\section{Effect on graft-versus-host disease}

As shown in Figure 5, cumulative incidences of acute GVHD grade II to IV and grade III to IV were evaluated in all studies for 633 patients and three of four studies for 438 patients, respectively. The grade II to IV aGVHD were significantly less frequent in the RIC group (OR 0.62; 95\% CI 0.44-0.87; $\mathrm{P}=0.006$ ) compared with the MAC group. There was no statistically significant heterogeneity among the trials $\left(\mathrm{I}^{2}=0 \%\right.$ ). Regarding more severe aGVHD (grades III to IV), there was a non-significant trend between the two intensities. (OR 0.58; 95\% CI 0.32-1.05; $\mathrm{P}=0.07$ ) with low heterogeneity among the trials $\left(\mathrm{I}^{2}=42 \%\right)$. At the end of follow-up, the cumulative incidence of overall and extensive chronic GVHD was obtained in four and three studies, respectively. There was evidence for significantly lower rates of overall cGVHD in the RIC arm (OR 0.71; 95\% CI 0.51-1.00, P=0.05) with moderate heterogeneity $\left(\mathrm{I}^{2}=50 \%\right)$. However, the available evidence from three trials including 508 patients was not sufficiently powered to show a statistically significant difference in extensive cGVHD between the two intervention groups (OR 1.12; 95\% CI $0.72-1.76, \quad \mathrm{P}=0.61)$ with extremely low $\left(\mathrm{I}^{2}=0 \%\right)$ heterogeneity.

\section{Subgroup analyses focusing on AML}

Treatment effects were evaluable for 450 patients with AML from three of four trials [21, 23, 24]. As presented in Figure 6, OS and PFS were not significantly different between the two intensity regimens, with pooled OR of 0.88 (95\% CI 0.34-2.26, $\mathrm{P}=0.79)$ and $1.16 \quad(95 \% \quad \mathrm{CI} \quad 0.41-3.25, \quad \mathrm{P}=0.78)$, respectively. Besides, the comprehensive analyses from two trials [21, 23] indicated a potential improvement in NRM and grade II-IV aGVHD for RIC compared with MAC (OR 0.59; 95\% CI 0.30-1.14, $\mathrm{P}=0.12$ for NRM, OR 0.59; $95 \%$ CI 0.31-1.11, $\mathrm{P}=0.10$ for grade II-IV aGVHD), and the incidences of relapse and overall cGVHD were comparable at the end of the study. Furthermore, in risk stratification by cytogenetics, two studies $[23,24]$ reported comparable outcomes for intermediate-risk patients, while the smallest RCT [21] indicated better PFS in the RIC group. For high-risk patients with AML, Scott et al. [24] claimed worse OS with RIC, whereas Rinden et al. [21] showed a PFS benefit and Bornhäuser et al. presented no significant difference in OS, PFS, RI, NRM between two arms (Table 2). 
RIC MAC Odds Ratio Odds Ratio

A Study or Subgroup Events Total Events Total Weight M-H, Random, $95 \% \mathrm{Cl} \quad \mathrm{M}-\mathrm{H}, \mathrm{Random}, 95 \% \mathrm{Cl}$

1.1.1 OS at 1 year

Bornhäuser 2018

Kroger 2018

Ringden 2013

Scott 2020

Subtotal $(95 \% \mathrm{Cl})$

$\begin{array}{lll}\text { Total events } & 69 & 72 \\ \text { Heterogeneity: } \mathrm{Tau}^{2}=0.39 ; \mathrm{Chi}^{2}=9.57, \mathrm{df}=3(\mathrm{P}=0.02) ; \mathrm{I}^{2}=69 \%\end{array}$

$\begin{array}{lllll}19 & 99 & 26 & 96 & 8.4 \%\end{array}$

$\begin{array}{lllll}11 & 65 & 17 & 64 & 6.9 \%\end{array}$

$\begin{array}{lllll}1 & 18 & 5 & 19 & 1.8 \%\end{array}$

$\begin{array}{rrrrr}38 & 137 & 24 & 135 & 9.3 \% \\ & 319 & & 314 & 26.3 \%\end{array}$

Test for overall effect: $Z=0.68(P=0.50)$

1.1.2 OS at 2 years

Bornhäuser 2018

Kroger 2018

Ringden 2013

Scott 2020

Subtotal $(95 \% \mathrm{Cl})$

$\begin{array}{lllll}27 & 99 & 34 & 96 & 9.0 \% \\ 15 & 65 & 24 & 64 & 7.6 \%\end{array}$

$\begin{array}{rrrrr}3 & 18 & 6 & 19 & 3.2 \%\end{array}$

$\begin{array}{ll}53 \quad 137 \\ & 319\end{array}$

$\begin{array}{lll}39 & 135 & 10.0 \%\end{array}$

Total events $98 \quad 103$

Heterogeneity: $\mathrm{Tau}^{2}=0.24 ; \mathrm{Chi}^{2}=8.32, \mathrm{df}=3(\mathrm{P}=0.04) ; \mathrm{I}^{2}=64 \%$

Test for overall effect: $Z=0.76(P=0.45)$

1.1.3 OS at 4 years

Bornhäuser 2018

Ringden 2013

Scott 2020

Subtotal $(95 \% \mathrm{Cl})$

Total events

$\begin{array}{rrrrr}36 & 99 & 43 & 96 & 9.3 \%\end{array}$

$\begin{array}{rrrrr}4 & 18 & 7 & 19 & 3.6 \% \\ 70 & 137 & 47 & 135 & 10.2 \%\end{array}$

$\begin{array}{lll}47 \quad 135 & 10.2 \%\end{array}$

$\begin{array}{lll}254 & 250 \quad 23.1 \%\end{array}$

$0.64[0.33,1.25]$

$0.56[0.24,1.32]$

$0.16[0.02,1.58]$

$1.78[1.00,3.17]$

$0.76[0.35,1.66]$

Heterogeneity: $\mathrm{Tau}^{2}=0.41 ; \mathrm{Chi}^{2}=8.64, \mathrm{df}=2(\mathrm{P}=0.01) ; \mathrm{I}^{2}=77 \%$

Test for overall effect: $Z=0.01(P=0.99)$

1.1.4 OS at 5 years

Bornhäuser 2018

Kroger 2018

Ringden 2013

Subtotal $(95 \% \mathrm{Cl})$

Total events

$\begin{array}{lllll}36 & 99 & 44 & 96 & 9.3 \%\end{array}$

$8.3 \%$

$0.70[0.40,1.25]$

$0.49[0.11,2.09]$

$1.96[1.20,3.18]$

$0.99[0.42,2.35]$

$0.50[0.23,1.08]$

$0.43[0.09,2.09]$

$0.78[0.42,1.46]$

Heterogeneity: $\mathrm{Tau}^{2}=0.00 ; \mathrm{Chi}^{2}=0.46, \mathrm{df}=2(\mathrm{P}=0.79) ; \mathrm{I}^{2}=0 \%$

Test for overall effect: $Z=2.40(P=0.02)$

Total $(95 \% \mathrm{Cl})$

Total events

$337^{1074} 353$

$1057 \quad 100.0 \%$

Heterogeneity: $\mathrm{Tau}^{2}=0.20 ; \mathrm{Chi}^{2}=33.18, \mathrm{df}=13(\mathrm{P}=0.002) ; \mathrm{I}^{2}=61 \%$

Test for overall effect: $Z=1.41(P=0.16)$

Test for subaroun differences: $\mathrm{Chi}^{2}=1.40 . \mathrm{df}=3(\mathrm{P}=0.70), \mathrm{I}^{2}=0 \%$

RIC

MAC

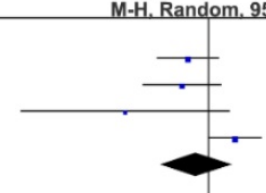

Odds Ratio

$0.50[0.25,1.03]$

$0.59[0.39,0.91]$

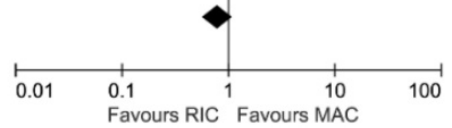

B Study or Subgroup Events Total Eyents Total Weight M-H Random. 95\% 1.4.1 PFS at 1 year Bornhäuser 2018

Kroger 2018

Scott 2020

Subtotal $(95 \% \mathrm{CI})$

Total events

$\begin{array}{lllll}30 & 99 & 32 & 96 & 9.7 \%\end{array}$

$\begin{array}{lllll}19 & 65 & 20 & 64 & 8.7 \%\end{array}$

$\begin{array}{lllll}68 & 137 & 31 & 135 & 10.2 \%\end{array}$

$117 \quad 83$

Heterogeneity: $\mathrm{Tau}^{2}=0.57 ; \mathrm{Chi}^{2}=13.52, \mathrm{df}=2(\mathrm{P}=0.001) ; \mathrm{I}^{2}=85 \%$

Test for overall effect: $Z=0.72(P=0.47)$

1.4.2 PFS at 2 years

Bornhäuser 2018

Kroger 2018

Scott 2020

Subtotal $(95 \% \mathrm{Cl})$

Total events

$\begin{array}{lllll}36 & 99 & 41 & 96 & 9.9 \%\end{array}$

$\begin{array}{lllll}25 & 65 & 27 & 64 & 9.0 \%\end{array}$

$\begin{array}{lllll}79 & 137 & 45 & 135 & 10.4 \%\end{array}$

.

$0.87[0.48,1.59]$

$0.91[0.43,1.93]$

$3.31[1.96,5.58]$

$1.41[0.55,3.56]$

Heterogeneity: $\mathrm{Tau}^{2}=0.49 ; \mathrm{Chi}^{2}=13.08, \mathrm{df}=2(\mathrm{P}=0.001) ; \mathrm{I}^{2}=85 \%$

Test for overall effect: $Z=0.48(P=0.63)$

1.4.3 PFS at 4 years

Bornhäuser 2018

Scott 2020

Subtotal $(95 \% \mathrm{Cl})$

Total events

$\begin{array}{lllll}41 & 99 & 47 & 96 & 9.9 \%\end{array}$

$0.74[0.42,1.30]$

$0.77[0.43,1.36]$

$0.86[0.42,1.73]$

$2.72[1.66,4.46]$

$1.24[0.52,2.94]$

Heterogeneity: $\mathrm{Tau}^{2}=0.77 ; \mathrm{Chi}^{2}=11.56, \mathrm{df}=1(\mathrm{P}=0.0007) ; \mathrm{I}^{2}=91 \%$

Test for overall effect: $Z=0.54(P=0.59)$

1.4.4 PFS at 5 years

Bornhäuser 2018

Kroger 2018

Ringden 2013

Subtotal $(95 \% \mathrm{Cl})$

Total events

$\begin{array}{lllll}41 & 99 & 47 & 96 & 9.9 \%\end{array}$

$9.9 \%$

$0.74[0.42,1.30]$

(1.65, 4.43$]$

$1.42[0.40,5.09]$

Heterogeneity: $\mathrm{Tau}^{2}=0.00 ; \mathrm{Chi}^{2}=0.13, \mathrm{df}=2(\mathrm{P}=0.94) ; \mathrm{I}^{2}=0 \%$

Test for overall effect: $Z=1.17(P=0.24)$

Total $(95 \% \mathrm{Cl})$

Total events 460

$1020 \quad 1000 \quad 100.0 \%$

$0.81[0.40,1.61]$

$1.06[0.13,8.47]$

$0.77[0.50,1.19]$

Odds Ratio

Heterogeneity: $\mathrm{Tau}^{2}=0.35 ; \mathrm{Chi}^{2}=46.14, \mathrm{df}=10(\mathrm{P}<0.00001) ; \mathrm{I}^{2}=78 \%$

Test for overall effect: $Z=0.91(P=0.36)$

Test for subaroun differences: $C^{2}{ }^{2}=2.30 . d f=3(P=0.51), I^{2}=0 \%$

M-H, Random, 95\% Cl

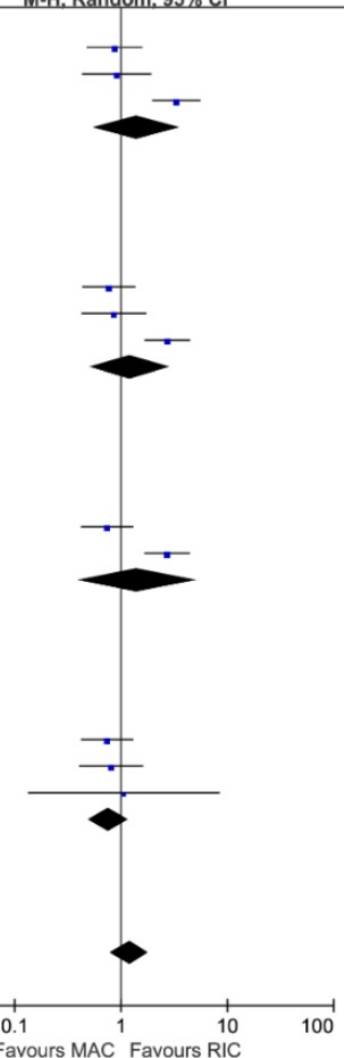

Figure 3. Forest plots for (A) Overall survival (OS) and (B) Progression-free survival at $1 / 2 / 4 / 5$ years. 


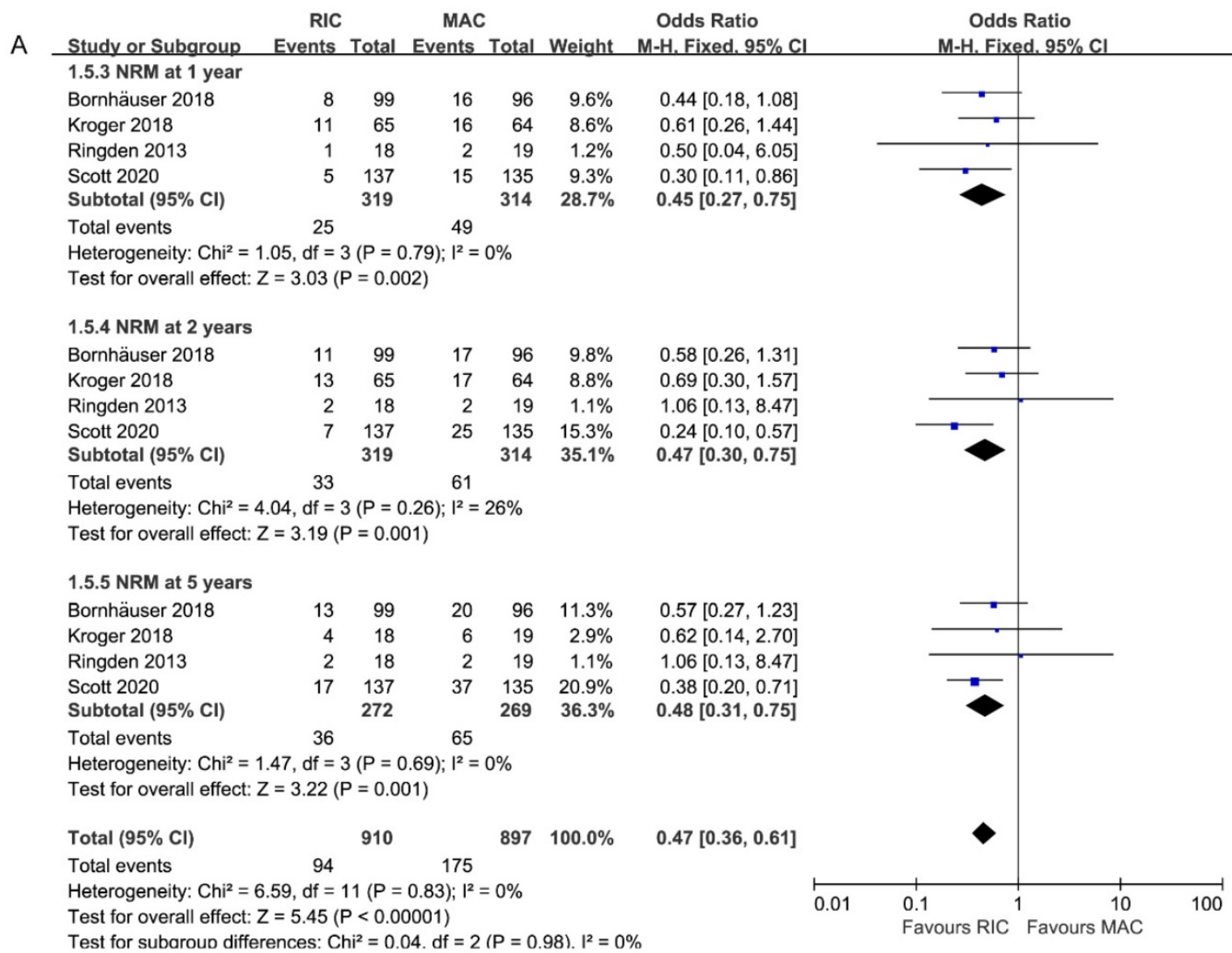

$$
\text { RIC MAC Odds Ratio Odds Ratio }
$$

B Study or Subgroup Events Total Events Total Weight M-H, Random, $95 \% \mathrm{Cl} \quad \mathrm{M}-\mathrm{H}$, Random, $95 \% \mathrm{Cl}$

1.7.3 RI at 1 year

Bornhäuser 2018

Kroger 2018

Ringden 2013

Scott 2020

Subtotal $(95 \% \mathrm{Cl})$

Total events

$\begin{array}{rrrrr}22 & 99 & 16 & 96 & 9.6 \% \\ 8 & 65 & 4 & 64 & 7.4 \% \\ 1 & 18 & 3 & 19 & 4.0 \%\end{array}$

$\begin{array}{lllll}1 & 18 & 3 & 19 & 4.0 \%\end{array}$

$\begin{array}{lllll}63 & 137 & 14 & 135 & 9.9 \%\end{array}$

Heterogeneity: $\mathrm{Tau}^{2}=1.00 ; \mathrm{Chi}^{2}=15.32, \mathrm{df}=3(\mathrm{P}=0.002) ; \mathrm{I}^{2}=80 \%$

Test for overall effect: $Z=1.28(P=0.20)$

1.7.4 RI at 2 years

Bornhäuser 2018

Kroger 2018

Ringden 2013

Scott 2020

Subtotal $(95 \% \mathrm{Cl})$

Total events

$\begin{array}{rr}25 & 99 \\ 11 & 65 \\ 2 & 18 \\ 71 & 137 \\ & 319\end{array}$

$\begin{array}{llll}99 & 24 & 96 & 9.9 \%\end{array}$

$$
\begin{array}{lll}
10 & 64 & 8.7 \%
\end{array}
$$

$\begin{array}{lll}5 & 19 & 5.5 \%\end{array}$

$20 \quad 135 \quad 10.1 \%$

Heterogeneity: $\mathrm{Tau}^{2}=1.21 ; \mathrm{Chi}^{2}=23.82, \mathrm{df}=3(\mathrm{P}<0.0001) ; \mathrm{I}^{2}=87 \%$

Test for overall effect: $Z=0.59(P=0.56)$

1.7.5 RI at 5 years

Bornhäuser 2018

Kroger 2018

Ringden 2013

Scott 2020

Subtotal $(95 \% \mathrm{Cl})$

Total events

Heterogeneity: $\mathrm{Tau}^{2}=1.09 ; \mathrm{Chi}^{2}=24.06, \mathrm{df}=3(\mathrm{P}<0.0001) ; \mathrm{I}^{2}=88 \%$

Test for overall effect: $Z=0.42(P=0.68)$

$\begin{array}{llllll}\text { Total }(95 \% \mathrm{Cl}) & 957 & 942 & 100.0 \% & 1.60[0.89,2.90]\end{array}$

Heterogeneity: $\mathrm{Tau}^{2}=0.82 ; \mathrm{Chi}^{2}=65.37, \mathrm{df}=11(\mathrm{P}<0.00001) ; \mathrm{I}^{2}=83 \%$

Test for overall effect: $Z=1.56(P=0.12)$

Test for subaroun differences: $\mathrm{Chi}^{2}=0.42 . \mathrm{df}=2(\mathrm{P}=0.81) . \mathrm{I}^{2}=0 \%$

$1.43[0.70,2.92]$

$2.11[0.60,7.38]$

$0.31[0.03,3.34]$

$7.36[3.85,14.06]$

$2.11[0.67,6.67]$

$1.01[0.53,1.94]$ $1.10[0.43,2.80]$ $0.35[0.06,2.10]$ $6.19[3.46,11.06]$ $1.43[0.43,4.70]$

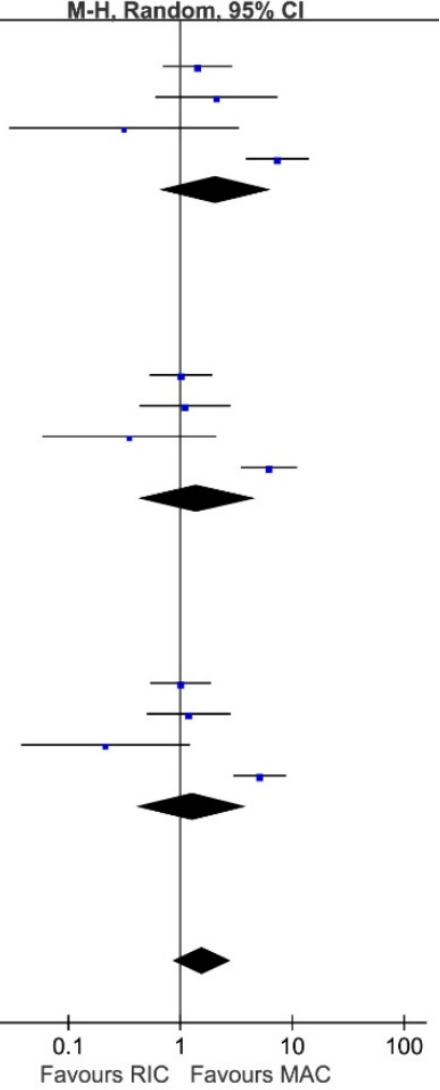

Figure 4. Forest plots for (A) non-relapse mortality (NRM) and (B) incidence of relapse (RI) at 1/2/5 years. 


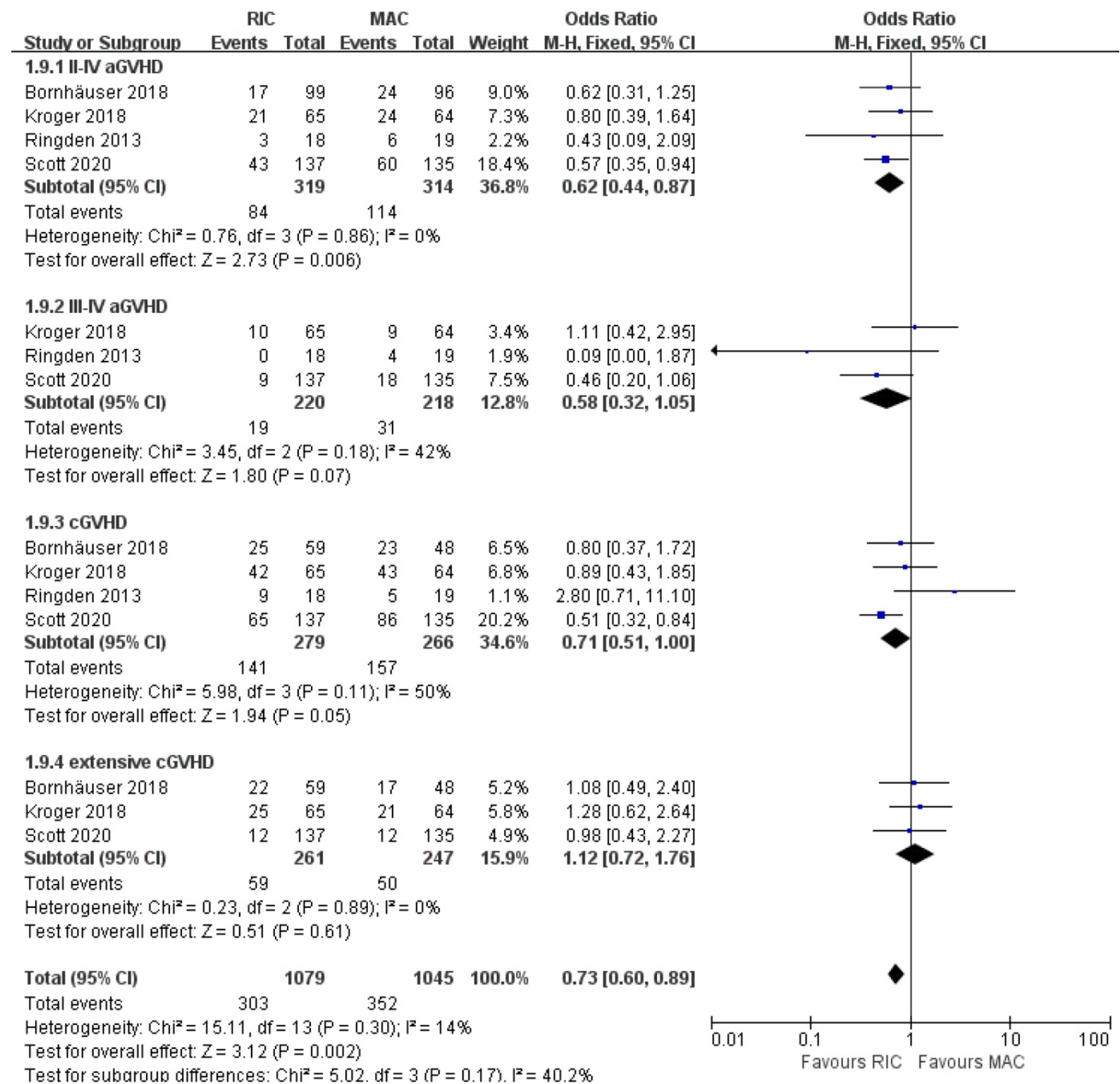

Figure 5. Forest plots for incidence of grade II-IV and grade III-IV acute graft-versus-host disease (aGVHD), overall and extensive chronic graft-versus-host disease (cGVHD) at the end of studies.

Table 2. Disease status, cytogenetic risk stratification and outcomes for acute myeloid leukemia

\begin{tabular}{llllll}
\hline & Disease status & Risk stratification & RIC & MAC & Outcomes \\
\hline Bornhäuser 2018 & CR1; $<5 \%$ marrow myeloblasts pre-HSCT & Intermediate§ & 77 & 70 & No significant difference in OS, PFS, RI, NRM \\
& & High & 22 & 26 & No significant difference in OS, PFS, RI, NRM \\
Rinden 2013 & CR1 or CR2 & Intermediate & 11 & 12 & 3-year PFS was better in RIC group than in MAC group (90\% vs. 75\%) \\
& & High & 3 & 3 & 3-year PFS was better in RIC group than in MAC group (67\% vs. 0\%) \\
Scott 2020* & CR; $<5 \%$ marrow myeloblasts pre-HCT & Intermediate† & 71 & 74 & No significant difference in OS \\
& & High & 61 & 54 & MAC was associated with a significant OS benefit.
\end{tabular}

RIC, reduced-intensity conditioning; MAC, myeloablative conditioning; CR, complete remission; OS, overall survival; PFS, progression-free survival; RI, relapse incidence; NRM, non-relapse mortality.

§Intermediate=normal and non-high risk, including normal karyotype and other intermediate abnormalities.

IIncluding the following cytogenetic abnormalities: +8; Complex ( $\geq 3$ aberrations); $-5,-7, \operatorname{del}(5 \mathrm{q}) ; \operatorname{Inv}(3), \mathrm{t}(3 ; 3) ; \mathrm{t}(6 ; 11), \mathrm{t}(11 ; 19) ; \mathrm{t}(6 ; 9)$.

†Defined according to the Eastern Cooperative Oncology Group/SWOG cytogenetic classification schema.

‡Including unfavorable risk cytogenetics according to the Eastern Cooperative Oncology Group/SWOG, FLT3 mutation regardless of accompanied cytogenetic risk.

*Data are for patients with acute myeloid leukemia and myelodysplastic syndromes combined. 


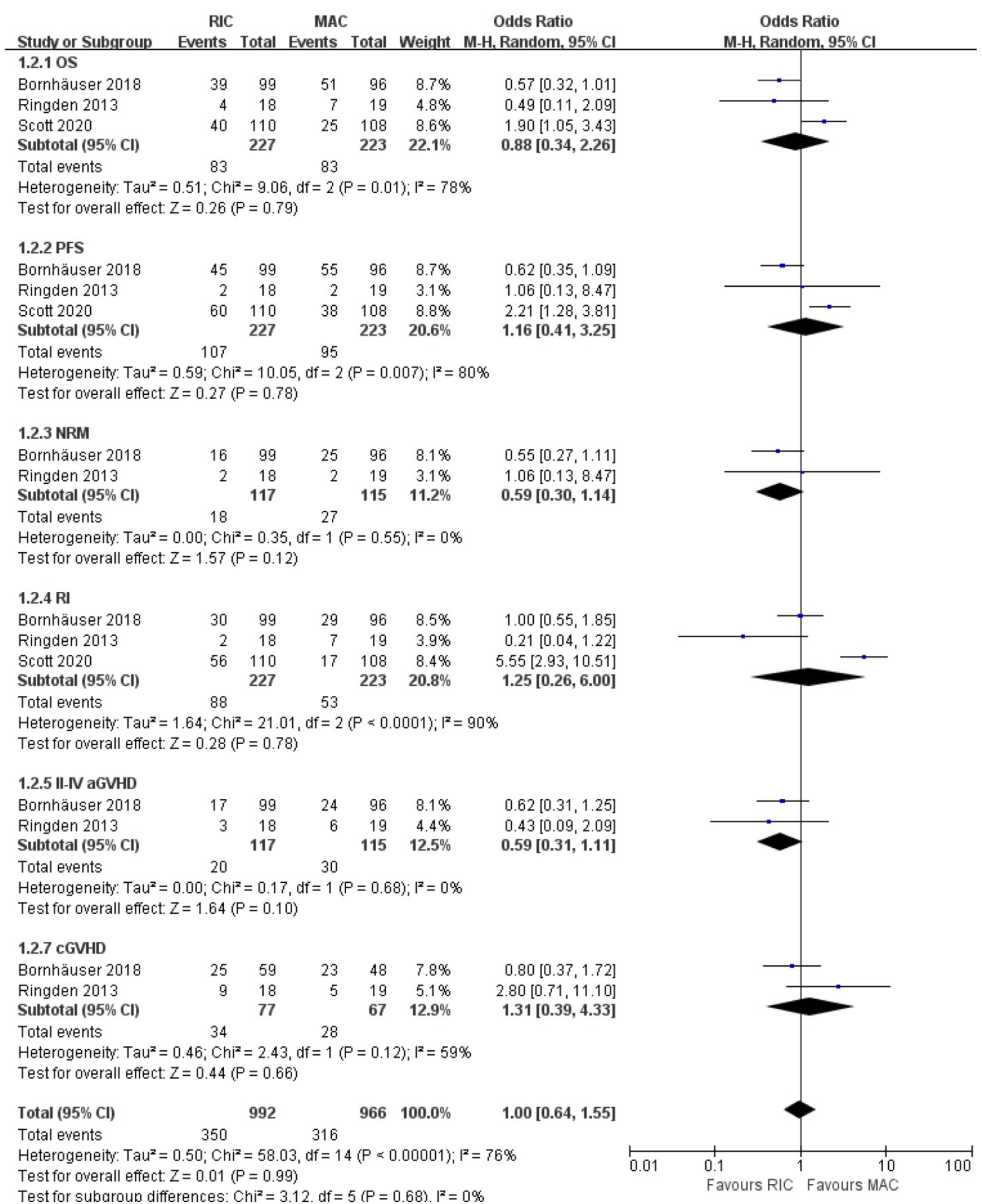

Figure 6. Forest plots for the subset of patients with acute myeloid leukemia. OS, overall survival; PFS, progression-free survival; NRM, non-relapse mortality; RI, relapse incidence; aGVHD, acute graft-versus-host disease; cGVHD, chronic graft-versus-host disease.

\section{Subgroup analyses focusing on MDS}

As shown in Figure 7, two out of four trials involving 183 patients with MDS reported OS, PFS, and relapse incidence. Likewise, none of the pooled results was statistically significant between the two conditioning intensities. For NRM, aGVHD and cGVHD, one study [22] provided similar effects in two arms, whereas the other [24] only reported them for AML and MDS patients as a whole, suggesting 
better outcomes in RIC regimens. The trial conducted by Kroger et al. [22] stratified cytogenetic risks into three levels and indicated that RIC in the low-risk cytogenetic group resulted in lower NRM but similar NRM in intermediate- and high-risk groups. In the BMT CTN 0901 [24], no significant difference was found in OS for standard-risk patients, while RIC in the high-risk cytogenetic group resulted in worse OS (Table 3).

\section{Discussion}

In this systematic review, we included all high-quality RCTs and yielded two main conclusions:
Regarding toxicity, we noted that RIC was associated with a lower risk of NRM and GVHD. Besides, the survival and relapse rates of the two regimens were comparable. Although not all studies present five-year data, we have found that the RIC group had a better five-year OS. For subset analyses of AML patients, we found that RIC had similar survival and relapse risk compared with MAC, with a trend towards less NRM and grade II-IV GVHD in RIC regimens. For patients with MDS, available data indicated comparable outcomes in OS, PFS and relapse.

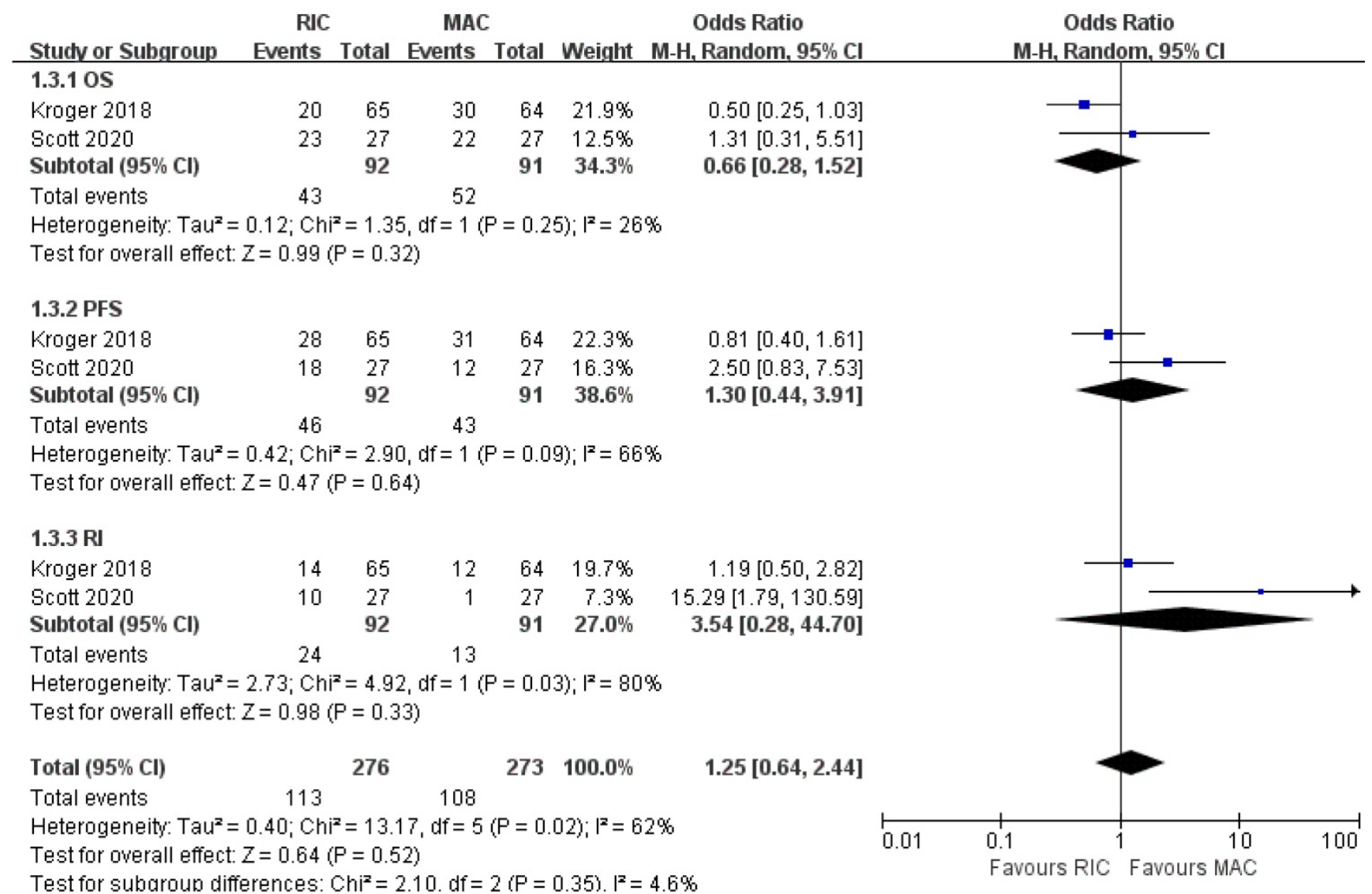

Figure 7. Forest plots for the subset of patients with myelodysplastic syndrome. OS, overall survival; PFS, progression-free survival; RI, relapse incidence.

Table 3. Disease status, cytogenetic risk stratification and outcomes for myelodysplastic syndrome

\begin{tabular}{|c|c|c|c|c|c|}
\hline & Disease status & Risk stratification & RIC & MAC & Outcomes \\
\hline \multirow[t]{3}{*}{ Kroger 2018} & MDS: 115 ; sAML: $12 ;$ missing: $2 ;<20 \%$ marrow myeloblasts pre-HCT & Low & 28 & 24 & RIC resulted in significantly lower NRM \\
\hline & & Intermediate & 13 & 17 & No significant difference in NRM \\
\hline & & High & 18 & 17 & No significant difference in NRM \\
\hline \multirow[t]{2}{*}{ Scott $2020^{*}$} & $<5 \%$ marrow myeloblasts pre-HCT & Intermediate & 71 & 74 & No significant difference in OS \\
\hline & & High $\ddagger$ & 61 & 54 & MAC was associated with a significant OS benefit \\
\hline
\end{tabular}

RIC, reduced-intensity conditioning; MAC, myeloablative conditioning; CR, complete remission; MDS, myelodysplastic syndrome; sAML, secondary acute myeloid leukemia; OS, overall survival; NRM, non-relapse mortality.

fintermediate-II or high-risk disease per the International Prognostic Scoring System.

${ }^{*}$ Data are for patients with acute myeloid leukemia and myelodysplastic syndromes combined. 
The risk bias of published RCTs was evaluated as low to moderate with randomized settings. Avoiding the selection bias and recalling bias, this meta-analysis provides the highest current level of evidence for the question in hand for AML and MDS patients. Although previous systematic reviews [30-32] have reported the similar results as our metaanalysis except for higher relapse rate, such studies mainly included retrospective studies, in which RIC was generally used for older individuals while MAC for younger patients. Also, the individualized decisions of different attending physicians may inevitably interfere with the conclusions. The advantage of NRM and GVHD reminds us that the RIC regimen is a valuable choice for patients who cannot tolerate high-intensity conditioning [33-35]. A current retrospective study revealed that MAC improves outcomes for AML and MDS patients with relatively lower disease risk index (DRI) but has a similar impact on patients with higher DRI [36]. Nowadays, more and more novel strategies were being successfully used as subsequent maintenance therapy after allo-HSCT, which significantly reduced the recurrence of allo-HSCT recipients receiving RIC regimens [37-39]. Additionally, the long-term GVHD/ relapse-free survival was also comparable in two arms for AML patients [40-42] but superior in RIC arm for MDS patients [43].

For AML patients, RIC regimens have demonstrated at least non-inferior survival and a trend of less toxicity. A sizeable observational analysis by the EBMT focusing on 2974 middle-aged (40-60) patients with AML [44] demonstrated that RIC resulted in higher OS and comparable RI in low-risk AML. However, the OS was similar and RI was higher in the intermediate- or high-risk patients. Additionally, the NRM was lower in all three cytogenetic risk groups, which was consistent with our findings in Figure 4A. From another perspective, patients in CR1 with high-risk cytogenetics or with MRD positive in genomics or multi-parameter flow cytometry (MFC) can benefit more from the MAC regimens [45-47].

As for the effect on MDS patients, we took account of the updated reports with longer follow-up $[25,26]$ and yielded results consistent with the previous studies [22, 24, 48]. Furthermore, we systematically reviewed that RIC resulted in less NRM in low-risk groups [22] but better OS in high-risk groups [24] for MDS patients. Besides, the genomics of MDS might have the potential to influence the optimal selection of conditioning intensity. CIBMTR research revealed a higher relapse rate in MDS patients with RAS pathway mutations only after RIC. In contrast, conditioning intensity didn't make a difference in outcomes for TP53-mutated MDS [49].

Of note, only one included clinical trial published by Scott and colleagues [26] reported worse outcomes of RIC. By contrast, the other three RCTs supported RIC for at least similar survival and less toxicity. Though the most weight was assigned to study by Scott BL et al. in the meta-analysis because of its largest sample size, there was still no evidence that reduced-intensity conditioning was inferior to MAC in either AML or MDS. Further sensitivity analysis confirmed the robustness of the results. There are several possible sources of heterogeneity. It was noteworthy that the trial by Scott et al. was the only one that included patients beyond CR2 and applied multiple choices of conditioning regimens and GVHD prophylaxis regimens in each intensity arm. Besides, it could conceivably be hypothesized that the heterogeneity could partially derive from the different distributions of cytogenetic risks and potential MRD in included RCTs.

Although stringent criteria were applied to identify and include studies for our meta-analysis, inherently, like any meta-analysis, there are some limitations in our study. Firstly, although stringent criteria have been applied in our meta-analysis, the baseline characteristics were impractical to be unified among studies. For example, one of the studies [21] has only enrolled 18 and 19 patients in two arms, respectively. The study published by Scott et al. included patients beyond CR2, while others restricted to the first or second CR. Secondly, the limited number of RCTs may have an impact on the statistical power of our results. Finally, regimens of different intensities were not completely uniform in included RCTs, though we tried to decrease the bias through heterogeneity analysis and got reliable conclusions.

Our results indicate that RIC is not inferior to MAC for AML patients with comparable posttransplantation survival, relapse risk, as well as potential advantages in NRM and GVHD. For MDS patients, neither survival nor relapse rate was significantly different. Based on the above results, it might be concluded that RIC is a feasible treatment option for adults with AML or MDS younger than 66 years, particularly those with intermediate-risk disease. Future RCTs incorporating of risk stratifications are warranted to guide the optimal decision under certain conditions.

\section{Acknowledgments}

This study was funded by the National Natural Sciences Foundation of China (Grant No. 81302043, $81500109,81500168)$ and the Major International Joint Research Project of China (No.31620103909). We 
thank Liangkai Chen from the School of Public Health, Tongji Medical College, Huazhong University of Science and Technology for statistics advice.

\section{Author Contributions}

Shengling $\mathrm{Ma}$ and Wei Shi designed the systematic review. Shengling $\mathrm{Ma}$ and Ziying $\mathrm{Li}$ performed the search and study selection. Linghui Xia, Liang Tang, and Huafang Wang extracted and analyzed the data. Shengling Ma and Wei Shi drafted the manuscript. $\mathrm{Yu} \mathrm{Hu}$ took responsibility for the whole process.

\section{Competing Interests}

We declare that we have no financial and personal relationships with other people or organizations that can inappropriately influence our work, there is no professional or other personal interest of any nature or kind in any product, service and/or company that could be construed as influencing the position presented in, or the review of, the manuscript entitled, "Reduced-intensity versus myeloablative conditioning regimens for younger adults with acute myeloid leukemia and myelodysplastic syndrome: a systematic review and meta-analysis".

\section{References}

1. Gratwohl A, Baldomero H, Aljurf M, Pasquini MC, Bouzas LF, Yoshimi A, et al. Hematopoietic stem cell transplantation: a global perspective. Jama. 2010; 303: 1617-24.

2. Tallman MS, Wang ES, Altman JK, Appelbaum FR, Bhatt VR, Bixby D, et al. Acute Myeloid Leukemia, Version 3.2019, NCCN Clinical Practice Guidelines in Oncology. Journal of the National Comprehensive Cancer Network. 2019; 17: 721-49.

3. Lim Z, Brand R, Martino R, van Biezen A, Finke I, Bacigalupo A, et al. Allogeneic hematopoietic stem-cell transplantation for patients 50 years or older with myelodysplastic syndromes or secondary acute myeloid leukemia. Journal of clinical oncology : official journal of the American Society of Clinical Oncology. 2010; 28: 405-11.

4. McClune BL, Weisdorf DJ, Pedersen TL, Tunes da Silva G, Tallman MS, Sierra $\mathrm{J}$, et al. Effect of age on outcome of reduced-intensity hematopoietic cell transplantation for older patients with acute myeloid leukemia in first complete remission or with myelodysplastic syndrome. Journal of clinical oncology : official journal of the American Society of Clinical Oncology. 2010; 28: 1878-87.

5. Sebert M, Porcher R, Robin M, Ades L, Boissel N, Raffoux E, et al. Equivalent outcomes using reduced intensity or conventional myeloablative conditioning transplantation for patients aged 35 years and over with AML. Bone marrow transplantation. 2015; 50: 74

6. Flynn CM, Hirsch B, DeFor T, Barker JN, Miller JS, Wagner JE, et al. Reduced intensity compared with high dose conditioning for allotransplantation in acute myeloid leukemia and myelodysplastic syndrome: a comparative clinical analysis. American journal of hematology. 2007; 82: 867-72.

7. Scott B, Sandmaier B, Storer B, Maris M, Sorror M, Maloney D, et al. Myeloablative vs nonmyeloablative allogeneic transplantation for patients with myelodysplastic syndrome or acute myelogenous leukemia with multilineage dysplasia: a retrospective analysis. Leukemia. 2006; 20: 128

8. Massenkeil G, Nagy M, Neuburger S, Tamm I, Lutz C, Le Coutre P, et al. Survival after reduced-intensity conditioning is not inferior to standard high-dose conditioning before allogeneic haematopoietic cell transplantation in acute leukaemias. Bone marrow transplantation. 2005; 36: 683

9. Alyea EP, Kim HT, Ho V, Cutler C, DeAngelo DJ, Stone R, et al. Impact of conditioning regimen intensity on outcome of allogeneic hematopoietic cell transplantation for advanced acute myelogenous leukemia and myelodysplastic syndrome. Biology of Blood and Marrow Transplantation. 2006; 12: 1047-55.

10. Pérez-Simón JA, Díez-Campelo M, Martino R, Brunet S, Urbano Á, Caballero $\mathrm{MD}$, et al. Influence of the intensity of the conditioning regimen on the characteristics of acute and chronic graft-versus-host disease after allogeneic transplantation. British journal of haematology. 2005; 130: 394-403.

11. Ringdén O, Labopin M, Ehninger G, Niederwieser D, Olsson R, Basara N, et al. Reduced intensity conditioning compared with myeloablative conditioning using unrelated donor transplants in patients with acute myeloid leukemia. Journal of Clinical Oncology. 2009; 27: 4570-7.

12. NCCN. NCCN Clinical Practice Guidelines in Oncology. (NCCN Guidelines ${ }^{\circledR}$ ) for Acute Myeloid Leukemia (Version 3.2020, December 23, 2019).

13. Group GW. Grading quality of evidence and strength of recommendations. BMJ: British Medical Journal. 2004; 328: 1490.

14. Higgins JP, Green S. Cochrane Handbook for Systematic Reviewsof Interventions Version 5.1. 0 (updated March 2011). The Cochrane Collaboration, 2011. Available from www cochrane-handbook org. 2017.

15. Moher D, Liberati A, Tetzlaff J, Altman DG. Preferred reporting items for systematic reviews and meta-analyses: the PRISMA statement. Annals of internal medicine. 2009; 151: 264-9.

16. Higgins JP, Altman DG, Gøtzsche PC, Jüni P, Moher D, Oxman AD, et al. The Cochrane Collaboration's tool for assessing risk of bias in randomised trials. Bmj. 2011; 343: d5928.

17. Tierney JF, Stewart LA, Ghersi D, Burdett S, Sydes MR. Practical methods for incorporating summary time-to-event data into meta-analysis. Trials. 2007; 8: 16.

18. Parmar MK, Torri V, Stewart L. Extracting summary statistics to perform meta-analyses of the published literature for survival endpoints. Statistics in medicine. 1998; 17: 2815-34.

19. Higgins JP, Thompson SG, Deeks JJ, Altman DG. Measuring inconsistency in meta-analyses. Bmi. 2003; 327: 557-60.

20. DerSimonian R, Laird N. Meta-analysis in clinical trials. Controlled clinical trials. 1986; 7: 177-88.

21. Ringden O, Erkers T, Aschan J, Garming-Legert K, Le Blanc K, Hagglund H, et al. A prospective randomized toxicity study to compare reduced-intensity and myeloablative conditioning in patients with myeloid leukaemia undergoing allogeneic haematopoietic stem cell transplantation. Journal of internal medicine 2013 : 274: 153-62.

22. Kroger N, Iacobelli S, Franke G-N, Platzbecker U, Uddin R, Hubel K, et al. Dose-Reduced Versus Standard Conditioning Followed by Allogeneic Stem-Cell Transplantation for Patients With Myelodysplastic Syndrome: A Prospective Randomized Phase III Study of the EBMT (RICMAC Trial). Journal of clinical oncology : official journal of the American Society of Clinical Oncology. 2017; 35: 2157-64.

23. Bornhauser M, Kienast J, Trenschel R, Burchert A, Hegenbart U, Stadler M, et al. Reduced-intensity conditioning versus standard conditioning before allogeneic haemopoietic cell transplantation in patients with acute myeloid leukaemia in first complete remission: a prospective, open-label randomised phase 3 trial. The Lancet Oncology. 2012; 13: 1035-44.

24. Scott BL, Pasquini MC, Logan BR, Wu J, Devine SM, Porter DL, et al. Myeloablative Versus Reduced-Intensity Hematopoietic Cell Transplantation for Acute Myeloid Leukemia and Myelodysplastic Syndromes. Journal of clinical oncology : official journal of the American Society of Clinical Oncology. 2017; 35: 1154-61.

25. Kroeger N, Iacobelli S, Koster L, Niederwieser D, Platzbecker U, Huebel K, et al. Reduced Intensity Vs. Myeloablative Conditioning Followed By Allogeneic Stem Cell Transplantation for Patients with Myelodysplastic Syndrome: Long Term Follow-up of a Prospective Randomized EBMT Phase III Study (RICMAC-Trial). Blood. 2018; 132: 1019-

26. Scott BL. Long-Term Follow up of BMT CTN 0901, a Randomized Phase III Trial Comparing Myeloablative (MAC) to Reduced Intensity Conditioning (RIC) Prior to Hematopoietic Cell Transplantation (HCT) for Acute Myeloid Leukemia (AML) or Myelodysplasia (MDS)(MAvRIC Trial). Biology of Blood and Marrow Transplantation. 2020; 26: S11.

27. Fasslrinner F, Schetelig J, Burchert A, Kramer M, Trenschel R, Hegenbart U, et al. Long-term efficacy of reduced-intensity versus myeloablative conditioning before allogeneic haemopoietic cell transplantation in patients with acute myeloid leukaemia in first complete remission: retrospective follow-up of an open-label, randomised phase 3 trial. The Lancet Haematology. 2018; 5: e161-e9.

28. Lioure B, Bene MC, Pigneux A, Huynh A, Chevallier P, Fegueux N, et al. Early matched sibling hematopoietic cell transplantation for adult AML in first remission using an age-adapted strategy: long-term results of a prospective GOELAMS study. Blood. 2012; 119: 2943-8.

29. Sébert $\mathrm{M}$, Porcher $\mathrm{R}$, Robin $\mathrm{M}$, Adès $\mathrm{L}$, Boissel $\mathrm{N}$, Raffoux $\mathrm{E}$, et al. Equivalent outcomes using reduced intensity or conventional myeloablative conditioning transplantation for patients aged 35 years and over with AML. Bone marrow transplantation. 2015; 50: 74-81.

30. Zeng W, Huang L, Meng F, Liu Z, Zhou J, Sun H. Reduced-intensity and myeloablative conditioning allogeneic hematopoietic stem cell transplantation in patients with acute myeloid leukemia and myelodysplastic syndrome: a meta-analysis and systematic review. International journal of clinical and experimental medicine. 2014; 7: 4357-68

31. Abdul Wahid SF, Ismail NA, Mohd-Idris MR, Jamaluddin FW, Tumian N, Sze-Wei EY, et al. Comparison of reduced-intensity and myeloablative conditioning regimens for allogeneic hematopoietic stem cell transplantation in patients with acute myeloid leukemia and acute lymphoblastic leukemia: a meta-analysis. Stem Cells Dev. 2014; 23: 2535-52. 
32. Zhang Z-H, Lian X-Y, Yao D-M, He P-F, Ma J-C, Xu Z-J, et al. Reduced intensity conditioning of allogeneic hematopoietic stem cell transplantation for myelodysplastic syndrome and acute myeloid leukemia in patients older than 50 years of age: a systematic review and meta-analysis. Journal of cancer research and clinical oncology. 2017; 143: 1853-64.

33. Cornelissen JJ, van Putten WLJ, Verdonck LF, Theobald M, Jacky E, Daenen SMG, et al. Results of a HOVON/SAKK donor versus no-donor analysis of myeloablative HLA-identical sibling stem cell transplantation in first remission acute myeloid leukemia in young and middle-aged adults: benefits for whom? Blood. 2007; 109: 3658-66.

34. Burnett AK, Wheatley K, Goldstone AH, Stevens RF, Hann IM, Rees JHK, et al. The value of allogeneic bone marrow transplant in patients with acute myeloid leukaemia at differing risk of relapse: results of the UK MRC AML 10 trial. British journal of haematology. 2002; 118: 385-400.

35. Martino R, de Wreede L, Fiocco M, van Biezen A, von dem Borne PA, Hamladji RM, et al. Comparison of conditioning regimens of various intensities for allogeneic hematopoietic SCT using HLA-identical sibling donors in AML and MDS with $<10 \%$ BM blasts: a report from EBMT. Bone marrow transplantation. 2013; 48: 761-70.

36. Solh MM, Solomon SR, Morris LE, Zhang X, Holland HK, Bashey A. The Dilemma of Conditioning Intensity: When Does Myeloablative Conditioning Improve Outcomes for Allogeneic Hematopoietic Cell Transplantation. Biology of Blood and Marrow Transplantation. 2019; 25: 606-12.

37. Platzbecker U, Middeke JM, Sockel K, Herbst R, Wolf D, Baldus CD, et al. Measurable residual disease-guided treatment with azacitidine to prevent haematological relapse in patients with myelodysplastic syndrome and acute myeloid leukaemia (RELAZA2): an open-label, multicentre, phase 2 trial. The Lancet Oncology. 2018; 19: 1668-79.

38. DiNardo CD, Stein EM, de Botton S, Roboz GJ, Altman JK, Mims AS, et al. Durable Remissions with Ivosidenib in IDH1-Mutated Relapsed or Refractory AML. The New England journal of medicine. 2018; 378: 2386-98.

39. Stone RM, Mandrekar SJ, Sanford BL, Laumann K, Geyer S, Bloomfield CD, et al. Midostaurin plus Chemotherapy for Acute Myeloid Leukemia with a FLT3 Mutation. The New England journal of medicine. 2017; 377: 454-64.

40. Baron F, Ruggeri A, Beohou E, Labopin M, Sanz G, Milpied N, et al. RIC versus MAC UCBT in adults with AML: a report from Eurocord, the ALWP and the CTIWP of the EBMT. Oncotarget. 2016; 7: 43027.

41. Shimoni A, Labopin M, Savani B, Volin L, Ehninger G, Kuball J, et al. Long-term survival and late events after allogeneic stem cell transplantation from HLA-matched siblings for acute myeloid leukemia with myeloablative compared to reduced-intensity conditioning: a report on behalf of the acute leukemia working party of European group for blood and marrow transplantation. Journal of hematology \& oncology. 2016; 9: 118.

42. Han LJ, Wang Y, Fan ZP, Huang F, Zhou J, Fu YW, et al. Haploidentical transplantation compared with matched sibling and unrelated donor transplantation for adults with standard-risk acute lymphoblastic leukaemia in first complete remission. British journal of haematology. 2017; 179: 120-30.

43. Park S-S, Jeon Y-W, Min GJ, Park S, Yahng S-A, Yoon J-H, et al, Graft-versus-Host Disease-Free, Relapse-Free Survival after Allogeneic Stem Cell Transplantation for Myelodysplastic Syndrome. Biology of Blood and Marrow Transplantation. 2019; 25: 63-72.

44. Passweg JR, Labopin M, Cornelissen J, Volin L, Socié G, Huynh A, et al. Conditioning intensity in middle-aged patients with AML in first CR: no advantage for myeloablative regimens irrespective of the risk group-an observational analysis by the Acute Leukemia Working Party of the EBMT. Bone marrow transplantation. 2015; 50: 1063-8.

45. Konuma T, Kondo T, Mizuno S, Doki N, Aoki J, Fukuda T, et al. Conditioning Intensity for Allogeneic Hematopoietic Cell Transplantation in Acute Myeloid Leukemia Patients with Poor-Prognosis Cytogenetics in First Complete Remission. Biology of Blood and Marrow Transplantation. 2020; 26: 463-71.

46. Hourigan CS, Dillon LW, Gui G, Logan BR, Fei M, Ghannam J, et al. Impact of Conditioning Intensity of Allogeneic Transplantation for Acute Myeloid Leukemia With Genomic Evidence of Residual Disease. Journal of clinical oncology : official journal of the American Society of Clinical Oncology. 2019; JCO1903011.

47. Ustun C, Courville EL, DeFor T, Dolan M, Randall N, Yohe S, et al. Myeloablative, but not reduced-intensity, conditioning overcomes the negative effect of flow-cytometric evidence of leukemia in acute myeloid leukemia. Biology of Blood and Marrow Transplantation. 2016; 22: 669-75.

48. Rashidi A, Meybodi MA, Cao W, Chu H, Warlick ED, Devine S, et al. Myeloablative versus Reduced-Intensity Hematopoietic Cell Transplantation in Myelodysplastic Syndromes: Systematic Review and Meta-analysis. Biology of blood and marrow transplantation : journal of the American Society for Blood and Marrow Transplantation. 2020.

49. Lindsley RC, Saber W, Mar BG, Redd R, Wang T, Haagenson MD, et al. Prognostic Mutations in Myelodysplastic Syndrome after Stem-Cell Transplantation. The New England journal of medicine. 2017; 376: 536-47. 